\section{Inventario Concepción. Desarrollo y validación de un instrumento de tamizaje de depresión para atención primaria}

\author{
BENJAMÍN VICENTE, SANDRA SALDIVIA ${ }^{\mathrm{a}, \mathrm{b}}$, \\ ROBERTO MELIPILLÁN ${ }^{\mathrm{a}, \mathrm{c}}$, NAÍN HORMAZABAL, \\ ANA CARROZA, ROLANDO PIHAN ${ }^{\mathrm{b}}$
}

\section{Concepción inventory. Development of a screening instrument for depression in primary care in Chile}

Background: Screening instruments are required for the detection of depressive disorders by primary care practitioners. Aim: To develop a screening instrument to detect depression, based on data gathered interviewing patients attending primary health care settings. Material and Methods: The instrument was constructed with data about factors associated or triggering a depressive disorder obtained from 3,000 patients consulting for general morbidity. All patients answered the Composite International Diagnostic Interview, (version 2.1, section depression) and an inventory containing 39 risk factors for depression, obtained from the literature. A multiple imputation method using chained equations was carried out. Using a binary logistic regression with backward selection, an equation for depression screening was obtained. The c-index was calculated to estimate discriminating power of the model. A shrinkage factor was estimated to adjust the predictive model. Results: Estimations were carried out with data from 2,552 patients with a median age of 47 years ( $73 \%$ women). Fifty five percent lived with a partner and $45 \%$ had basic studies. The method selected 14 significant predictors, with a shrinkage value of 0.861 and a $c$-index of 0.838 (95\% confidence intervals 0.82-0.86). Conclusions: The instrument has adequate psychometric properties as a screening tool for depression in primary health care.

(Rev Med Chile 2016; 144: 555-562)

Key word: Depression; Diagnosis; Primary Health Care.
Departamento de Psiquiatría y Salud Mental, Universidad de Concepción. Concepción, Chile. aPsicólogo (a).

bPhD.

${ }^{\mathrm{C}} \mathrm{MsC}$.

Estudio financiado por el Proyecto FONDEF $\mathrm{N}^{\circ}$ DO2I-1140 y FONDECYT No 1110687.

Recibido el 12 de mayo de 2015 , aceptado el 7 de marzo de 2016.

Correspondencia a:

Benjamín Vicente

Casilla 160-C, Departamento

de Psiquiatría y Salud Mental,

Universidad de Concepción,

Concepción, Chile.

Tel-fax: 56-41-2312799

bvicent@udec.cl
S e ha comunicado una prevalencia de vida de trastorno depresivo mayor en América Latina entre 1,9 y $19,6 \%{ }^{1-13}$ y entre 4,7 y $29,5 \%$ en consultantes en centros de atención primaria en salud ${ }^{14}$. La brecha de atención varía de 43,4 a $78,2 \%{ }^{15}$. Esto es relevante, considerando que la depresión es responsable de $4,4 \%$ de años de vida saludable perdidos ajustados por discapacidad en el mundo, para todos los grupos etáreos, con gran impacto económico por costos directos y por las pérdidas productivas que determina ${ }^{16}$. Las personas que sufren esta enfermedad tienen peor calidad de vida, mayor riesgo de enfermar por otras causas y de morir ${ }^{16}$.

Los médicos no psiquiatras detectan la depresión en $36,4 \%$, entre personas que consultan por otras causas ${ }^{17}$. Para mejorar la detección se han realizado capacitaciones a los médicos, modalidad 
que ha mostrado ser poco efectiva ${ }^{18}$, siendo el uso de instrumentos de tamizaje una alternativa efectiva y de bajo costo.

Existen distintos instrumentos de tamizaje. El Patient Health Questionnaire (PHQ), en su versión de 9 preguntas (PHQ-9) tiene una sensibilidad de $0,8(95 \%$ CI $0,71-0,87)$ y una especificidad de 0,92, (95\% CI 0,88-0,95) $)^{19}$. Para la versión de 2 preguntas (PHQ-2) ha mostrado una sensibilidad de $0,81(0,75-0,84)$ y una especificidad de $0,76(0,74-0,78)^{20}$. La versión validada en Chile tiene una sensibilidad de 0,88 y una especificidad de $0,92^{21}$. El Cuestionario General de Salud de Goldberg tiene una sensibilidad de $76 \%$ y una especificidad de $73 \%{ }^{22}$, mientras que el Self Report Questionnaire (SRQ-20) tiene una sensibilidad y especificidad de $74 \%$ y $77 \%$, respectivamente ${ }^{22}$.

Guías clínicas estadounidenses y canadienses recomiendan el uso de instrumentos de tamizaje de depresión en atención primaria; en cambio, en el Reino Unido no, ya que no se ha encontrado evidencia de que pacientes sometidos a tamizaje tengan mejores resultados que quienes no se identifican de esta manera, cuando son tratados con las mismas terapias ${ }^{23}$.

En Chile no se dispone de estudios que permitan recomendar o rechazar la utilización de instrumentos de tamizaje. Sin embargo, los organismos gubernamentales han decidido su implementación en atención primaria ${ }^{24}$.

El objetivo de este estudio fue desarrollar un instrumento de tamizaje de depresión, de fácil aplicación a partir del estudio de una muestra de personas consultantes en atención primaria.

Los proyectos FONDEF y FONDECYT que hicieron posible el presente trabajo contaron con la aprobación del Comité de Ética de la Facultad de Medicina de la Universidad de Concepción.

\section{Material y Método}

Se trata de un estudio descriptivo, transversal, realizado en el marco de una investigación cuyo objetivo era desarrollar un índice de riesgo para predecir la incidencia de un episodio depresivo en pacientes que consultan en centros de atención primaria y que correspondió al estudio nacional del proyecto multicéntrico PREDICT-D ${ }^{25}$. Todos los usuarios fueron seguidos durante doce meses, con evaluaciones cada seis, para detectar los casos incidentes ${ }^{26,27}$.
Secundariamente, se identificó al conjunto de variables asociadas a la presencia de un episodio depresivo al inicio del estudio y se formuló un modelo para identificar la presencia de depresión en línea base, lo que permitió construir el instrumento de tamizaje aquí presentado, cuyo rendimiento fue posteriormente evaluado sobre la misma muestra en el seguimiento a los seis meses.

\section{Participantes}

Se estimó un tamaño muestral de 3.000 sujetos, suficiente para ser representativo de la población consultante y que permitiera alcanzar el poder estadístico necesario para dar validez al producto final de ese estudio. Se seleccionaron aleatoriamente diez centros de atención primaria en salud (APS) de la Provincia de Concepción. Se estimó el tamaño de muestra de cada uno y su distribución por sexo, en base en las características de la población atendida por cada dispositivo durante el año previo.

Fueron ingresadas al estudio personas de ambos sexos, de entre 15 y 75 años, que solicitaban atención espontánea por morbilidad general durante el último trimestre del año anterior. No se consideraron pacientes que acudían por consulta en programas específicos, sean crónicos u otros, a no ser que hubieran sido derivados desde el programa a consulta médica por alguna otra patología. Como criterio de exclusión se consideró la inhabilidad para entender el idioma local, dado que era un estudio multicéntrico, la presencia de psicosis, demencia o de una enfermedad física incapacitante, de acuerdo a lo registrado en la historia clínica del paciente.

\section{Procedimiento}

El proceso de selección contempló el ingreso de pacientes durante doce semanas, a partir del registro de usuarios a ser atendidos en cada centro cada día. Cada caso era seleccionado por un funcionario previamente capacitado, usando una tabla de números aleatorios. Identificado el usuario, se le asignaba un entrevistador, quien lo contactaba durante la semana y coordinaba la primera entrevista, cara a cara, en el mismo centro o en el hogar del entrevistado.

En esta entrevista el paciente era informado del estudio e invitado a participar. Si accedía, firmaba un formulario de consentimiento informado $y$, 
en ese momento, se aplicaban los instrumentos de evaluación.

Los entrevistadores, legos respecto de la clínica, recibieron previamente un curso de entrenamiento de 20 horas en el uso de los instrumentos. Al final de este proceso fueron evaluados y una proporción de ellos seleccionados como entrevistadores.

Todas las entrevistas fueron editadas, revisando que los aspectos formales y que la estructuración de saltos hubiera sido correctamente aplicada. Los eventuales errores fueron corregidos. Además, $10 \%$ de las entrevistas de cada entrevistador eran supervisadas en terreno, verificando que hubiese sido efectivamente realizada y que el procedimiento de aplicación hubiese sido correcto.

\section{Instrumentos}

A todos los participantes se les aplicó la Composite International Diagnostic Interview, versión 2.1 $(\mathrm{CIDI})^{28}$ sección Depresión, y un Inventario que recogía múltiples variables que la literatura sugería como eventuales factores protectores y de riesgo.

La Composite International Diagnostic Interview (CIDI), versión $2.1^{28}$ es una entrevista altamente estructurada que entrega diagnósticos psiquiátricos tanto actuales, como presentes a lo largo de la vida, de acuerdo a la Clasificación Internacional de Enfermedades en su décima edición (CIE-10 $)^{29}$ y al Manual Diagnóstico y Estadístico de los Trastornos Mentales, versión IV (DSMIV $)^{30}$, de la Asociación Psiquiátrica Americana. La validez de la CIDI frente al diagnóstico clínico es alta ${ }^{31}$. Se trata de un instrumento que ha sido traducido del inglés a muchos idiomas y que está validado en Chile, con reporte de kappa en valores que van de 0,52 para trastornos somatomorfos a 0,94 para trastornos afectivos ${ }^{32}$.

\section{Factores de riesgo}

Se recogió información de 39 factores de riesgo para construir un modelo usando regresión logística. Éstos cubrieron un amplio rango de potenciales variables relevantes sugeridas por la literatura:

1) Sociodemográficas: Edad; sexo; ocupación; nivel educacional; estado civil; situación de empleo; etnia; propiedad de la vivienda que ocupa; convivencia (vivir solo o con otros); nacimiento en el país de residencia o en el extranjero.
2) Satisfacción y nivel de demanda en su lugar de trabajo y en el hogar ${ }^{33}$.

3) Deudas y dificultades financieras.

4) Situación de salud actual: auto percepción de salud física y salud mental (Short Form $12)^{34}$; presencia de enfermedad física de larga evolución; consumo de alcohol en los 6 meses previos a la entrevista (AUDIT) ${ }^{35}$; síntomas de ansiedad y pánico en los seis meses previos $(\mathrm{PHQ})^{36}$.

5) Antecedentes clínicos y psicológicos: Antecedentes de depresión en la vida (CIDI) ${ }^{28}$; existencia de problemas con el alcohol o antecedentes de tratamiento asociado a su consumo; uso de otras drogas durante la vida; experiencias infantiles de abuso físico o emocional; o de abuso sexual.

6) Antecedentes clínicos y psicológicos en la familia: Presencia de problemas graves de salud física, psicológica, de consumo de drogas, o discapacidades graves en personas cercanas al paciente; problemas psicológicos serios; $y$ antecedentes de suicidio en familiares de primer $\operatorname{grado}^{37}$.

7) Variables relacionales y de apoyo social: Calidad de las relaciones sexuales; y calidad de la relación emocional con la pareja o esposo(a); dificultades en iniciar y mantener relaciones interpersonales cercanas; y la percepción de apoyo social de familiares y amigos ${ }^{38}$.

8) Percepción de estrés asociado a una ocupación, sea empleo remunerado o no, en los seis meses previos a la entrevista, incluyó la percepción de control en la actividad laboral, el experimentar dificultades y no percibir apoyo y experimentar malestar por el no reconocimiento de la tarea realizada.

9) Grado de satisfacción con el barrio en que vive; y percepción de seguridad experimentada dentro y fuera de la residencia.

10)Ocurrencia de eventos vitales en los 6 meses previos a la entrevista ${ }^{39}$.

11)Creencias religiosas o espirituales.

12)Experiencias de discriminación en el período, derive ésta del sexo, edad, etnia, apariencia, discapacidad u orientación sexual.

\section{Análisis estadístico}

Todos los análisis se llevaron a cabo empleando el programa Stata 12.1. Debido a la presencia de valores ausentes en algunas de las variables, se 
utilizó imputación múltiple mediante ecuaciones encadenadas, implementado en el comando mi impute chained de Stata. Se imputaron 50 conjuntos de datos, sobre cada uno de los cuales se llevaron a cabo los análisis estadísticos, cuyos resultados fueron combinados mediante las reglas de Rubin ${ }^{40}$ para obtener los resultados finales del estudio.

Para obtener la ecuación para el screening de depresión, sobre cada uno de los 50 conjuntos de datos imputados, se utilizó una adaptación del método de selección con propósito de Hosmer y cols ${ }^{41}$. Se realizó una selección inicial de predictores relevantes del diagnóstico de depresión, mediante análisis de regresión logístico univariados de cada variable independiente, de los cuales se seleccionaron aquellos con valor $\mathrm{p}<0,20$. En el caso de las variables con múltiples alternativas de nivel ordinal, se dicotomizó la variable de acuerdo a los coeficientes de cada categoría en la regresión logística que estuviesen más cercanos al punto mínimo y máximo, considerando, además, que esa recodificación era consistente con una lógica clínica.

Se ajustó luego un modelo multivariado con todas las variables y se usó la técnica de backward selection, consistente en eliminar secuencialmente las variables cuyos coeficientes fueran menores y que tuvieran valor $\mathrm{p}>0,05$, hasta que todos los coeficientes fuesen estadísticamente significativos. Las variables edad y género fueron incluidas en todos los modelos de regresión debido a su conocida asociación con la variable dependiente.

Para evaluar el desempeño del modelo final se ocupó el c-index, también conocido como el área bajo la curva ROC, que representa la probabilidad promedio del modelo para discriminar un eventual caso positivo de depresión de uno negativo. Para lograr una predicción con menor varianza cuando se estima el riesgo en nuevas muestras se usó el método propuesto por Copas ${ }^{42}$, que implicó estimar un factor de shrinkage que fue aplicado a los coeficientes del modelo.

\section{Resultados}

Tres mil pacientes fueron contactados, de ellos, 2.832 inicialmente aceptaron participar. Sin embargo, sólo 2.552 participantes proporcionaron un número suficiente de respuestas para ser incluidos en los análisis.
Las características sociodemográficas de la muestra nacional se presentan en la Tabla 1. Como se observa, predominan las mujeres $(72,8 \%)$, los casados o que viven en pareja $(55,1 \%)$, con estudios básicos (45,4\%), estudios medios $(45,9 \%)$ y una edad media de 47,4 años.

De los 48 predictores considerados, 45 fueron seleccionados para el análisis multivariado. El análisis de regresión logístico multivariado empleando el método backward selection permitió identificar 14 predictores que resultaron estadísticamente significativos en el nivel $\alpha=0,05$ (Tabla 2). El valor del shrinkage aplicado a los coeficientes fue de 0,861 y el c-index del modelo fue 0,838 (95\% CI = 0,818-0,857).

Los puntajes de corte recomendados son: $\mathrm{Sin}$ depresión: menor o igual a -1,94; con riesgo de depresión: mayor a -1,94. Empleando este puntaje de corte se obtiene una sensibilidad de $82 \%$ y una especificidad de $73 \%$. El instrumento final se presenta en la Tabla 3.

Tabla 1. Características sociodemográficas de la muestra

\begin{tabular}{|lrr|}
\hline \multicolumn{2}{|c|}{ n } & \multicolumn{1}{c|}{$\%$} \\
\hline Sexo & & \\
Masculino & 694 & 27,2 \\
Femenino & 1.858 & 72,8 \\
\hline Estado civil & & \\
Casado & 1.252 & 49,1 \\
Viviendo con su pareja o conviviendo & 155 & 6,1 \\
Separado/a de hecho & 209 & 8,2 \\
Viudo/a & 255 & 10,0 \\
Anulado/a & 11 & 0,4 \\
Soltero/a o nunca se ha casado/a & 670 & 26,3 \\
Escolaridad & & \\
Sin escolaridad & 102 & 4,0 \\
Educación básica & 1.159 & 45,4 \\
Educación media & 1.171 & 45,9 \\
Educación superior & 120 & 4,7 \\
\hline Total & \multicolumn{2}{|c}{} \\
Contactados & 3.000 & 100,0 \\
Incorporados & 2.832 & 94,4 \\
Analizados & 2.552 & 85,1 \\
\hline
\end{tabular}


Tabla 2. Coeficientes originales y coeficientes modificados del análisis de regresión logística

\begin{tabular}{|c|c|c|c|}
\hline & Coeficiente & SE & $\begin{array}{l}\text { Coeficiente después } \\
\text { corrección shrinkage }\end{array}$ \\
\hline Edad & $0,009 *$ & 0,004 & 0,007 \\
\hline Sexo masculino & $-0,464^{* *}$ & 0,161 & $-0,400$ \\
\hline Hacer menos de lo deseado por problemas emocionales & $1,151 * * *$ & 0,139 & 0,992 \\
\hline Sentirse calmado y tranquilo & $0,391 * *$ & 0,147 & 0,336 \\
\hline Tener mucha energía & $0,444^{* *}$ & 0,149 & 0,383 \\
\hline Sentirse desanimado o triste & $0,302^{*}$ & 0,145 & 0,260 \\
\hline Contar con familiares o amigos & $-0,467^{*}$ & 0,193 & $-0,402$ \\
\hline Presencia de discapacidad & $0,418^{* *}$ & 0,139 & 0,360 \\
\hline Robo o pérdida de un objeto de valor & $0,516^{*}$ & 0,224 & 0,445 \\
\hline Satisfacción con la situación de convivencia familiar & $0,131 *$ & 0,053 & 0,113 \\
\hline Crisis de ansiedad con miedo o pánico repentino & $0,830 * * *$ & 0,136 & 0,715 \\
\hline Percepción de discriminación por situación económica & $0,506^{* *}$ & 0,176 & 0,436 \\
\hline Satisfacción con la vida sexual & $0,405^{* *}$ & 0,133 & 0,349 \\
\hline Antecedentes de maltrato físico en la infancia & $0,383^{* *}$ & 0,143 & 0,330 \\
\hline Constante & $-3,990 * * *$ & 0,265 & $-3,436$ \\
\hline
\end{tabular}

${ }^{*} \mathrm{p}<0,05 ;{ }^{* *} \mathrm{p}<0,01 ;{ }^{* * *} \mathrm{p}<0,001$. Ha9r: recodificado de ha9 (1, 2, $\left.3->0 ; 4,5,6->1\right)$. Ha10r: recodificado de ha10 $(1,2,3->0 ; 4,5,6->1)$. Ha12r: recodificado de ha12 (1, 2, 3, $4->1 ; 5->0)$. Ff c: recodificado de ffc $(1,2->1 ; 3->0)$. Rel3r: recodificado de rel3 $(1,2,3->1 ; 4,5->0)$. Child1 r: recodificado de child1 $\overline{(1}, 2,3->0 ; 4,5->1)$.

\section{Tabla 3. Inventario Concepción para tamizaje de depresión}

1. Edad (en años cumplidos)

2. Sexo (marque según observe) Hombre $=1$

Mujer $=0$

3. Durante las últimas 4 semanas: ¿A causa de su salud emocional hizo menos de los que hubiera querido hacer?

Sí $=1$

$\mathrm{No}=0$

4. Durante las últimas 4 semanas: ¿Cuánto tiempo se sintió calmado y tranquilo?

Siempre $\quad=0$

Casi siempre $=0$

Muchas veces $=0$

Algunas veces $=1$

Sólo alguna vez $=1$

Nunca

$=1$

5. Durante las últimas 4 semanas: ¿Cuánto tiempo tuvo mucha energía?

Siempre $=0$

Casi siempre $=0$

Muchas veces $=0$

Algunas veces $=1$

Sólo alguna vez $=1$

Nunca

$=1$
6. Durante las últimas 4 semanas: ¿Cuánto tiempo se sintió desanimado y triste?
Siempre
$=0$
Casi siempre $=0$
Muchas veces $=0$
Algunas veces $=1$
Sólo alguna vez $=1$
Nunca
$=1$

7. Tengo familiares o amigos, con los que puedo contar pase lo que pase

$\begin{array}{ll}\text { Falso } & =1 \\ \text { A veces } & =1 \\ \text { Verdadero } & =0\end{array}$

8. Tiene alguna enfermedad, discapacidad o dolencia que le haya afectado o vaya a afectarle durante un período largo de tiempo

Sí $=1$

No $=0$

9. Durante los últimos 6 meses, ¿Le han robado o ha perdido algún objeto de valor?

Sí $=1$

No $=0$ 
Tabla 3. Inventario Concepción para tamizaje de depresión (continuación)

10. Si considera su situación en relación a con quién(es) comparte su vivienda en este momento, ¿Hasta qué punto está satisfecho con la situación que hay en su hogar?

Muy satisfecha $\quad=1$

Bastante satisfecha $\quad=2$

Ni satisfecha ni insatisfecha $=3$

Bastante insatisfecha $\quad=4$

Muy insatisfecha

$=5$

11 En los últimos 6 meses ¿ha tenido usted una crisis de ansiedad sintiendo miedo o pánico de repente?

Sí $=1$

No $=0$

12 En los últimos seis meses, ¿se ha sentido usted discriminado por su situación económica (pobreza, marginalidad)?

Sí $=1$

No $=0$

13 ¿Cómo considera su vida sexual en general?

Muy insatisfecha $\quad=1$

Moderadamente insatisfecha $=1$

Satisfecha e insatisfecha a la vez $=1$

Medianamente satisfecha $\quad=0$

Muy satisfecha

$=0$

14 Cuando era pequeño(a), ¿Cree que sufrió maltrato físico?

Nunca $\quad=0$

En contadas ocasiones $=0$

A veces $\quad=0$

A menudo $\quad=1$

Con frecuencia $\quad=1$

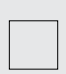

Interpretación de la puntuación: Una puntuación por sobre -1,94 refleja mayor probabilidad de presentar una enfermedad depresiva. Quienes obtienen puntuaciones por debajo de -1,94 tienen mayor probabilidad de no presentar una enfermedad depresiva.

\section{Discusión}

La baja capacidad de detección de depresión de los médicos no especialistas exige estrategias que permitan mejorar la pesquisa. El instrumento de tamizaje logra discriminar adecuadamente entre personas con o sin depresión, con resultados superiores a la capacidad de detección de médicos no psiquiatras informada en la literatura. Estos rendimientos son similares a otros tamizadores en estudios internacionales.

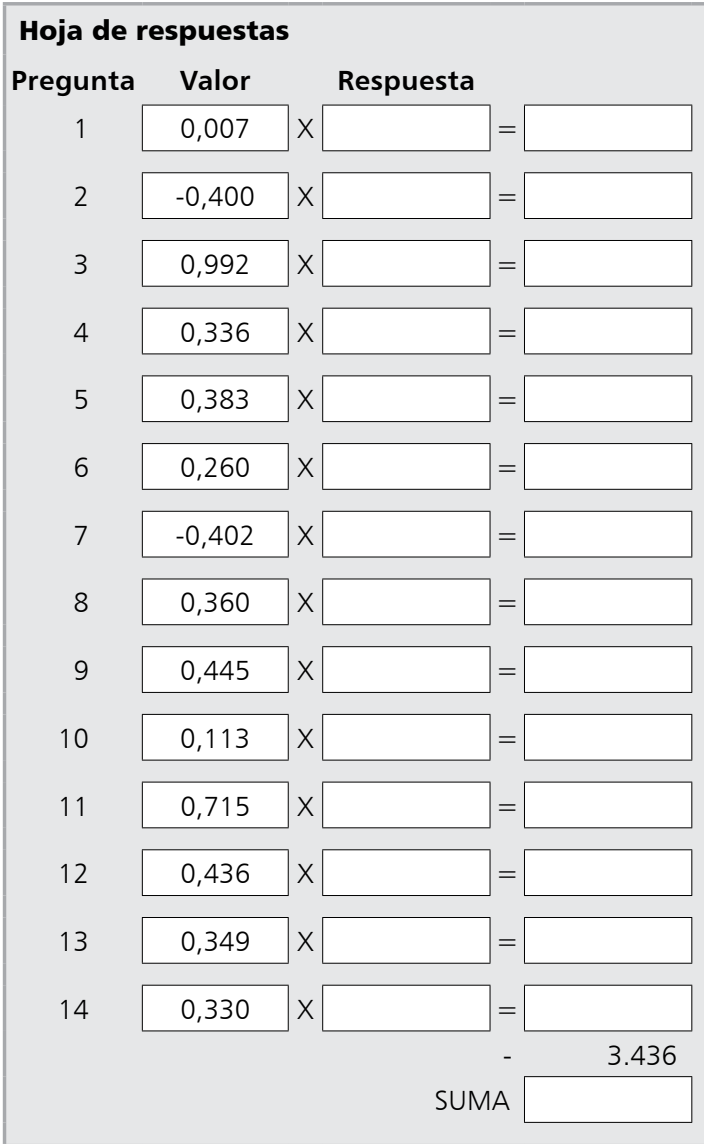

Puntaje de corte

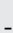

$-3$

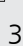

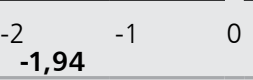


facilidad de aplicación e interpretación, así como la brevedad permiten señalar que es una buena alternativa para el tamizaje en atención primaria.

\section{Referencias}

1. Larraya F, Casullo M, Viola F. Prevalencia de la patología mental en la megalópolis de Buenos Aires. Buenos Aires: Consejo Nacional de Investigaciones Científicas y Técnicas. 1982.

2. Di Marco G. Prevalencia de desórdenes mentales en el área metropolitana de la República Argentina. Acta Psiquiatr Psicol Am Lat 1982; 28: 93-102.

3. Aszkenazi M, Casullo M. Factores socioculturales y presencia de psicopatologías en poblaciones de distintas localidades argentinas. Acta Psiquiatr Psicol Am Lat 1984; 30: 11-20.

4. Vorcaro C, Lima-Costa M, Barreto S, Uchoa E. Unexpected high prevalence of 1-month depression in a small Brazilian community: the Bambuí Study. Acta Psychiatr Scand 2011; 104: 257-63.

5. Almeida-Filho N, Mari J, Coutinho E, França J, Fernandes J, Andreoli S, et al. Estudo multicêntrico de morbidade psiquiátrica em áreas urbanas brasileiras (Brasília, São Paulo, Porto Alegre). Rev ABP-APAL 1992; 14: 93-104.

6. Almeida-Filho N, Mari J de J, Coutinho E, Franca J, Fernandes J, Andreoli S, et al. Brazilian multicentric study of psychiatric morbidity. Br J Psychiatry 1997; 171: 524-9.

7. Andrade L, Lolio C, Gentil V, Laurenti R. Epidemiologia dos transtornos mentais em uma área definida de captação da cidade de São Paulo, Brasil. Rev Psiquiatr Clín 1999; 26: 257-62.

8. Andrade L, Walters E, Gentil V, Laurenti R. Prevalence of ICD-10 mental disorders in a catchment area in the city of São Paulo, Brazil. Soc Psychiatry Psychiatr Epidemiol 2002; 37: 316-25.

9. Araya R, Rojas G, Fritsch R, Acuña J, Lewis G. Common mental disorders in Santiago, Chile: prevalence and socio-demographic correlates. Br J Psychiatry 2001; 178: 228-33.

10. Rioseco P, Escobar B, Vicente B, Vielma M, Saldivia S, Cruzat M, et al. Prevalencia de vida de algunos trastornos psiquiátricos en la provincia de Santiago. Rev Chil Neuro-Psiquiatr 1995; 11: 186-93.

11. Vicente B, Rioseco P, Vielma M, Uribe M, Boggiano G, Torres S. Prevalencia de vida de algunos trastornos psiquiátricos en la provincia de Concepción. Rev Chil de Neuro-Psiquiatr 1992; 9: 1050-60.
12. Vicente B, Kohn R, Rioseco P, Saldivia S, Baker C, Torres $\mathrm{S}$. Population prevalence of psychiatric disorders in Chile: 6-month and 1-month rates. Br J Psychiatry 2004; 184: 299-305.

13. Vicente B, Rioseco P, Saldivia S, Kohn R, Torres S. Estudio chileno de prevalencia de patología psiquiátrica (DSM-III-R/CIDI) (ECPP). Rev Med Chile 2002; 130: 527-36.

14. Goldberg D, Lecrubier Y. Form and frequency of mental disorders across centres. In: Üstün TB, Sartorius N, eds. Mental illness in general health care: an international study. Chichester, John Wiley \& Sons on behalf of WHO 1995; 323-34.

15. Kohn R, Levav I, Caldas de Almeida J, Vicente B, Andrade L, Caraveo-Anduaga J, et al. Los trastornos mentales en América Latina y el Caribe: asunto prioritario para la salud pública. Rev Panam Salud Publ 2005; 18 (4/5): 229-40.

16. Organización Mundial de la Salud. Informe sobre la salud en el mundo, la atención primaria de salud, más necesaria que nunca. OMS. 2008

17. Cepoiu M, McCusker J, Cole MG, Sewitch M, Belzile E, Ciampi A. Recognition of depression by non-psychiatric physicians-a systematic literature review and meta-analysis. J Gen Intern Med 2008; 23 (1): 25-36.

18. Levav I, Kohn R, Montoya I, Palacio C, Rozic P, Solano $\mathrm{I}$, et al. Training Latin American primary care physicians in the WPA module on depression: results of a multicenter trial, Psychological Medicine 2005; 35 (1): 35-45.

19. Gilbody S, Richards D, Brealey S, Hewitt C. Screening for depression in medical settings with the Patient Health Questionnaire (PHQ): a diagnostic meta-analysis. J Gen Intern Med 2007; 22 (11): 1596-602.

20. Zuithoff NP, Vergouwe Y, King M, Nazareth I, van Wezep MJ, Moons KG, et al. The Patient Health Questionnaire- 9 for detection of major depressive disorder in primary care: consequences of current thresholds in a cross sectional study. BMC Fam Pract 2010; 11: 98.

21. Baader T, Molina J, Venezian S, Rojas C, Farías R, Fierro-Freixenet $\mathrm{C}$, et al. Validación y utilidad de la encuesta PHQ-9 (Patient Health Questionnaire) en el diagnóstico de depresión en pacientes usuarios de atención primaria en Chile. Rev Chil Neuro-Psiquiatr. [revista en la Internet]. 2012.

22. Araya R, Wynn R, Lewis G. A comparison of two psychiatric case finding questionnaires (GHQ-20 and SRQ-20) in primary care in Chile. Social Psychiatry and Psychiatric Epidemiology 1992; 27: 168-73.

23. Thombs B, Coyne J, Cuijpers P. Rethinking recommendations for screening for depression in primary care. CMAJ 2012; 184. 
24. Ministerio de Salud de Chile. Guía Clínica Tratamiento de personas con depresión. Santiago: MINSAL. 2009.

25. King M, Weich S, Torres-González F, Svab I, Maaroos HI, Neeleman J, et al. Prediction of depression in European general practice attendees: the PREDICT study. N. V. 12-Jan-2006 In: BMC Public Health. 6, 8 p.6

26. King M, Walker C, Levy G, Bottomley C, Royston P, Weich S, et al. Development and validation of an international risk prediction algorithm for episodes of major depression in general practice attendees: the Predict D study. Archives of General Psychiatry 2008; 65 (12): 1368-1376. doi:10.1001/archpsyc.65.12.1368.

27. Saldivia S, Torres-González T, Runte-Geidel A, Xavier M, Grandon P, Antonioli C, et al. Standardization of the maristan scale of informal care in people with schizophrenia and other psychoses Acta Psiquiatrica Scandinava 2013; 1-7.

28. World Health Organisation (1997) Composite International Diagnostic Instrument (CIDI). Version 2.1 World Health Organisation, Geneva.

29. WHO. The CIE-10 Classification of Mental and Behavioural Disorders. WHO, Geneva, 1992.

30. American Psychiatric Association (APA). (1996). DSMIV. Barcelona: Masson.

31. Wittchen HU. Reliability and validity studies of the WHO-Composite International Diagnostic Interview (CIDI): a critical review. J Psychiatr Res 1994; 28: 57-84.

32. Vielma M, Vicente B, Rioseco P, Castro N, Torres S. Validación En Chile se una Entrevista Diagnóstica Estandarizada para Estudios Epidemiológicos CIDI. Revista de Psiquiatría 1992; 9 (1): 1039-49.

33. Karasek R, Theorell T, Schwartz JE, Schnall PL, Pieper $\mathrm{CF}$, Michela JL. Job characteristics in relation to the prevalence of myocardial infarction in the US health examination survey (HES) and the health and nutrition examination survey (HANES). Am J Public Health 1988; 78: 910-8.
34. Ware JE, Jr Harris WJ, Gandek B, Rogers BW, Reeses PR. MAP-R for windows: multitrait/multi-item analysis program-revised user's guide. Boston (MA): Health Assessment Laboratory; 1997.

35. Saunders J, Aasland O, Babor T, de la Fuente J, Grant M. Development of the Alcohol Use Disorders Identification Test (AUDIT): WHO Collaborative Project on Early Detection of Persons with Harmful Alcohol Consumption-II. Addiction 1993; 88: 791-804.

36. Kroenke K, Spitzer RL, Williams JBW. The PHQ-9: Validity of a brief depression severity measure. Journal of General Internal Medicine 2001; 16: 606-13.

37. Nurnberger JI, Jr Blehar MC, Kaufmann CA, York-Cooler C, Simpson SG, Harkavy-Friedman J, et al. Diagnostic interview for genetic studies. Rationale, unique features, and training. NIMH Genetics Initiative. Arch Gen Psychiatry 1994; 51 (11): 849-59; discussion 863-4.

38. Chaturvedi N, Sjolie AK, Stephenson JM, Abrahamian H, Keipes M, Castellarin A, et al. Effect of lisinopril on progression of retinopathy in normotensive people with type 1 diabetes. The EUCLID Study Group. EURODIAB Controlled Trial of Lisinopril in Insulin-dependent Diabetes Mellitus. Lancet 1998; 351: 28-31.

39. Brugha T, Bebbington P, Tennant C, Hurry J. The List of Threatening Experiences: a subset of 12 life event categories with considerable long-term contextual threat. Psychol Med 1985; 15 (1): 189-94.

40. Rubin DB. (1987) Multiple Imputation for Nonresponse in Surveys. New York: John Wiley and Sons. (2004) Classic edition.

41. Hosmer D, Lemeshow S, Sturdivant R. Applied logistic regression. 3a ed. New Jersey: John Wiley \& Sons, Inc.; 2013. 397 p.

42. Copas JB. Regression, prediction, and shrinkage. Journal of the Royal Statistical Society, Series B, Methodological 1983; 45: 311-54. 